\title{
Cervical spine imaging in trauma patients: a simple scheme of rationalising arm traction using zonal divisions of the vertebral bodies
}

\author{
D Ohiorenoya, M Hilton, C D H Oakland, C A J McLauchlan, M Cobby, A O Hughes
}

\begin{abstract}
Objective-To evaluate the effectiveness of arm traction for cervical spine imaging in trauma patients and devise a scheme to predict the probability of visualising the C7/T1 level in trauma patients.

Methods-98 trauma patients were studied. Each vertebral body was divided into three equal horizontal zones, the disc space between vertebral bodies being equivalent to one zone. The fifth cervical vertebra was used as the starting level (zone 1). Zones obtained pre and post arm traction on the lateral cervical spine radiographs were recorded. Results were analysed to show the probability of imaging the lower cervical spine, including the cervico-thoracic junction.
\end{abstract}

Results-If the initial film showed less than zone 10 (mid-C7 vertebra), the probability of showing zone 13 (upper body of T1) with arm traction was only $7 \cdot 7 \%$, that is, one success in every 13 pulls; or conversely, 12 failures in every 13 pulls.

Conclusions-Unless an initial cervical spine radiograph includes the upper one third of the body of the $\mathrm{C} 7$ vertebra, the probability of attaining the C7/T1 level with arm traction is $<15 \%$. It is suggested that all initial radiographs of the lateral cervical spine in major trauma patients be done with arm traction, and where the upper one third of the body of $\mathrm{C} 7$ vertebra is not seen, then computerised tomography, swimmer's, or oblique views be considered.

( Accid Emerg Med 1996;13:175-176)

Key terms: cervical spine radiography; trauma; arm-pull manoeuvre

Conventional radiographic imaging of the cervical spine including the first thoracic vertebra is an integral part of the initial assessment of patients who have sustained major trauma. A method employed to rule out injury to the cervical spine clearance in most accident and emergency $(A \& E)$ departments is arm traction. Although this method is widely used, the success rate in visualising the desired $\mathrm{C} 7 / \mathrm{T} 1$ level has never been evaluated, and some patients may therefore be subjected to arm traction where there is little chance of viewing the cervico-thoracic junction. As most missed lesions of the cervical spine occur at $\mathrm{C} 7 / \mathrm{T} 1$, it is essential that this junction is clearly visualised.
In this study we evaluate the effectiveness of the arm traction method, demonstrate its limitations, and devise a scheme to predict the probability of visualising the $\mathrm{C} 7 / \mathrm{T} 1$ level in trauma patients.

\section{Methods}

From March 1993 to February 1994, 98 trauma patients who had lateral cervical spine radiographs before and after arm traction were included in the study.

We divided each vertebral body into three equal horizontal zones (figure), with the disc space between vertebral bodies equivalent to one zone. The fifth cervical vertebra was used as the starting level (zone 1) because no patient in our study showed less than five cervical vertebral bodies before and after traction. Factors such as upper limb and shoulder girdle fractures and the Glasgow coma score, which might affect the efficacy of arm traction, were noted. Pre and post-traction radiographs were compared and the appropriate zones reported (figure).

Zones obtained before and after arm traction on the lateral cervical spine radiographs were recorded (table 1 ).

Analysis of the results showed the probabilities of imaging the lower cervical spine, including the cervico-thoracic junction.

\section{Results}

If the view included zone 9 (upper C7 vertebra) or less, the probability of showing zone 13 (upper body of T1) with arm traction was only

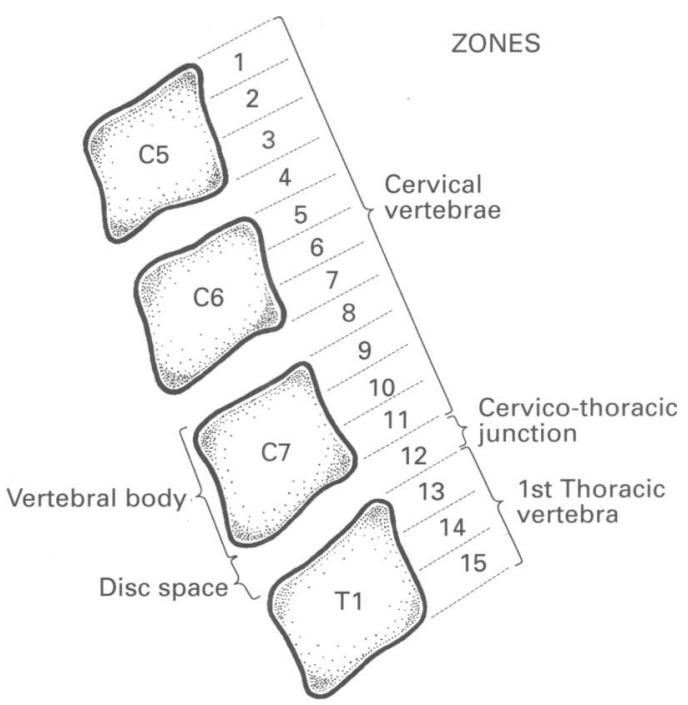

Zonal divisions of vertebral bodies (C5 to T1). 
Table 1 Zones obtained before and after arm traction on lateral cervical spine radiographs

\begin{tabular}{lllllllll}
\hline Zone after pull & \multicolumn{7}{l}{ Zone on initial lateral film } & \multirow{2}{*}{ Total } \\
\cline { 2 - 7 } & 6 or less & 7 & 8 & 9 & 10 & 11 & 12 & \\
\hline Zone 6 or less & 2 & - & - & - & - & - & - & 2 \\
Zone 7 & 2 & 0 & - & - & - & - & - & 2 \\
8 & 2 & 1 & 0 & - & - & - & - & 3 \\
9 & 0 & 1 & 3 & 0 & - & - & - & 4 \\
10 & 0 & 3 & 5 & 2 & 0 & - & - & 11 \\
11 & 0 & 0 & 2 & 6 & 1 & 2 & 1 & 21 \\
12 & 1 & 0 & 0 & 6 & 3 & 10 & 10 & 21 \\
13 (C7/T1 junction) & 0 & 0 & 1 & 2 & 10 & 9 & 7 & 8 \\
14 & 0 & 0 & 0 & 0 & 0 & 1 & 1 & 5 \\
15 & 0 & 0 & 0 & 0 & 1 & 3 & 19 & 98 \\
Total & 7 & 5 & 11 & 16 & 15 & 25 & 19 \\
\hline
\end{tabular}

Probability of showing zone 13.

If lateral film: 7 or less probability $=0 \%$

$\begin{array}{ll}7 \text { or less probability } & =0 \% \\ 8 & =9 \% \\ 9 & =12 \cdot 5 \% \\ 10 & =73 \% \\ 11 \text { or more } & =70 \%\end{array}$

Table 2 Glasgow coma score (GCS) and arm traction

- 82/98 patients had GCS of 15

- 7/98 patients had GCS of 14

- 9/98 patients had GCS of $<1$

( 2 were intubated and ventilated)

- Only $45 / 98$ showed $\geq 13$

$7 \cdot 7 \%$, that is, one success in every 13 pulls; or conversely, 12 failures in every 13 pulls. No correlation was found between the Glasgow coma score and the ease of applying arm traction (table 2).

\section{Discussion}

Manual arm traction remains the most popular means of demonstrating the lower cervical spine in most A\&E departments in the United Kingdom. Our study has shown that there is very little chance of success in achieving this objective if the initial film shows less than zone 10 (mid-C7 vertebra). This would also apply to patients who have arm traction routinely as part of the initial cervical spine $x$ rays. There would be no need to repeat the traction if zone 10 is not attained with the initial pull.

As most missed lesions of the cervical spine occur at the cervico-thoracic junction, ${ }^{12}$ that is, zones $12 / 13$, there is clearly a need to visualise this region by other imaging techniques such as swimmer's or oblique views, or a computerised tomography (CT) scan. The range of accuracy of a cross table lateral cervical spine $x$ ray has been estimated at between $70 \%$ and $90 \%$ by various investi- gators. ${ }^{13}$ One needs to correlate clinical and radiological findings in the initial management of these patients and protect the cervical spine appropriately.

Manual arm traction in most A\&E departments is a single operator manoeuvre. Various other methods have been described to clear the cervical spine. ${ }^{45}$ These include the use of traction devices and shoulder harness. ${ }^{5-7}$ The advantages these devices might have over manual arm traction are negated by the fact that they are impractical to use while active resuscitation is in progress.

CONCLUSIONS

(1) Arm traction is still the most widely used method for clearing the cervical spine in major trauma.

(2) Unless an initial cervical spine radiograph includes the upper one third of the body of $\mathrm{C} 7$ vertebra, the probability of viewing $\mathrm{C} 7 / \mathrm{T} 1$ level with arm traction is $<15 \%$.

(3) We advocate that all initial radiographs of the lateral cervical spine in major trauma patients be done with arm traction, and where the upper one third of the body of C7 vertebra is not seen, then a CT scan, swimmer's, or oblique views should be considered. (Only $20 \%$ of initial films without traction showed the C7/T1 level in our study.)

(4) In cases with upper limb fractures where arm traction may be difficult, and initial lateral cervical spine films inadequate, a CT scan may be required to show the lower cervical spine.

(5) Even with good quality lateral cervical spine films, $10 \%$ to $20 \%$ of soft tissue and bony abnormalities could still be missed.

We appreciate the assistance of Mrs Marlene Soden and Mr Phil Smith of the Accident and Emergency Radiology Department, Frenchay Hospital, and Mrs Jennie Beddoe for secretarial help.

Driscoll PA, Ross R. Nicholson DA. Cervical spine 1. BMJ 1993;ii:785-9.

2 Evans DK. Dislocations at the cervico-thoracic junction. f Bone foint Surg Br 1983;65B:124-7.

3 Hudgins PA, Hudgins RJ. Radiology of cervical spine trauma. Clin Neurosurg 1991;37:571-95.

4 Boger DC. Traction device to improve CT imaging of lower cervical spine. Am f Neuroradiol 1986;7:719-21.

5 Liebault B, Namour A, Cronier P, Talha A, Toulemande JL. How to visualise one additional vertebra on profile radiographs of the cervical spine. 7 Radiol 1989;70:507-9.

Gaylor AL, Sacksteder SP. A simple method of applying arn traction. Radiol Technol 1982;54:125-6.

7 Norris CS, Silva WE, Dodson TF, Darrow J. Shoulde ( cervical spine. Arch Surg 1986;121:452-5. 